\title{
Transanal Minimally Invasive Surgery
}

\author{
Earl V. Thompson, MD ${ }^{1}$ Joshua I. S. Bleier, MD ${ }^{1}$ \\ ${ }^{1}$ Department of Surgery, University of Pennsylvania Health System, \\ Philadelphia, Pennsylvania \\ Clin Colon Rectal Surg 2017;30:112-119.
}

\begin{abstract}
Address for correspondence Joshua I. S. Bleier, MD, FACS, FASCRS, Department of Surgery, University of Pennsylvania Health System, 800 Walnut St., 20th Floor, Philadelphia, PA 19106 (e-mail: Joshua.Bleier@uphs.upenn.edu).
\end{abstract}
Abstract
Keywords
- transanal endoscopic surgery
- transanal endoscopic microsurgery
- transanal minimally invasive surgery
- rectal cancer

Transanal endoscopic surgery (TES) techniques encompass a variety of approaches, including transanal endoscopic microsurgery and transanal minimally invasive surgery. These allow a surgeon to perform local excision of rectal lesions with minimal morbidity and the potential to spare the need for proctectomy. As understanding of the longterm outcomes from these procedures has evolved, so have the indications for TES. In this study, we review the development of TES, its early results, and the evolution of new surgical techniques. In addition, we evaluate the most recent research on indications and outcomes in rectal cancer.
Surgical management of rectal lesions can be challenging due to the anatomic restrictions of the bony pelvis and the morbidity associated with injury to nearby structures. Defining the balance between appropriate treatment of disease and patients' quality of life continues to drive the development of techniques and technology in this field. While radical resection of the entire rectum represents the best chance of cure for malignancy, it may also carry unacceptably high functional risks and morbidity for early-stage cancers that could be adequately treated by local resection. For early-stage cancers in the distal rectum, transanal excision (TAE) using traditional retractors is often sufficient, but visualization of the mid and proximal rectum is limited. More invasive techniques such as the transsacral (Kraske) and posterior transrectal (York-Mason) approaches offer better visualization, but their attendant complications such as fecal fistula or incontinence are formidable. As one of the earliest examples of natural orifice surgery, the introduction of transanal endoscopic microsurgery (TEM) was an elegant solution to these challenges, but, more than 30 years after the initial development, we continue to evaluate the best way to make use of these innovative techniques.

\section{History of Transanal Endoscopic Surgery}

The first transanal endoscopic surgery (TES) platform, TEM, developed in parallel with the rise of laparoscopic and minimally invasive abdominal surgery. The technique gained early adopters due to dissatisfaction with the outcomes of traditional TAE of rectal lesions but was slow to become widely accepted in part due to inexperience with minimally invasive abdominal surgery, as many surgeons became familiar with the two techniques simultaneously. Professor Gerhard Buess in Germany was the innovator behind the TEM operative proctoscope which allowed sufficient visualization and manipulation of rectal lesions beyond the reach of TAE. Professor Buess first published a description of his technique using animal models in the German literature in 1983, followed shortly thereafter by a series in the English literature describing the first 12 patients. ${ }^{1,2}$ In these initial reports, and still today, the $40-\mathrm{mm}$ diameter proctoscope with its beveled end is inserted using a blunt obturator and a sealing faceplate is applied to the outer orifice. The faceplate contains ports for the angled stereoscopic optic systems and working ports for surgical instruments. Binocular optics offer the surgeon depth perception via a three-dimensional view, unlike traditional laparoscopy. A novel insufflation system, coupled with suction and irrigation, provides a clear operative field. The entire TEM device is attached to the operating table using a support arm (-Fig. 1). After pneumorectum is established, suctioning is controlled via a roller-pump to prevent loss of insufflation.

The equipment and techniques have remained remarkably unchanged in the three decades since its initial description, including angled instruments and metal beads instead of tied suture. The most noticeable modification has been the
Issue Theme Laparoscopy, Endoscopy, and Minimally Invasive Colorectal Surgery; Guest Editor: James E. Duncan, MD, FACS, FASCRS.
Copyright $\odot 2017$ by Thieme Medical Publishers, Inc., 333 Seventh Avenue, New York, NY 10001, USA. Tel: +1(212) 584-4662.
DOI http://dx.doi.org/ 10.1055/s-0036-1597315. ISSN 1531-0043. 


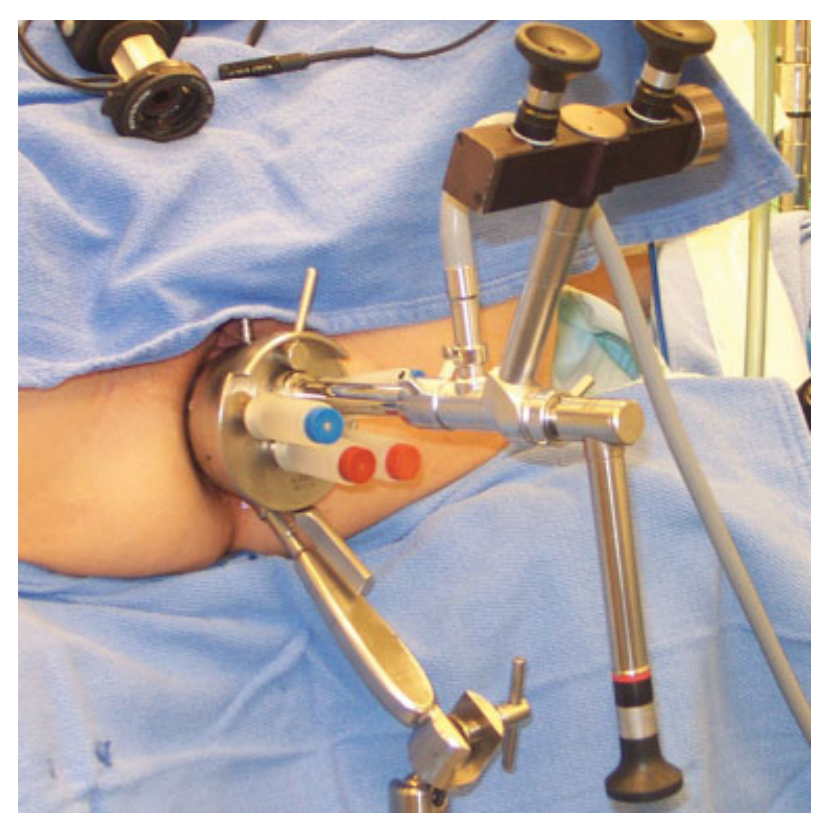

Fig. 1 The assembled TEM scope is shown. Note the upward facing stereoscopic eyepiece, downward facing monocular viewer for camera attachment, and support arm fixing the scope to the operating table in the center of the bottom of the image (Image courtesy of Dr. Peter Cataldo).

addition of an eyepiece for attachment of a laparoscopic camera, allowing for overhead viewing. As familiarity with the technique has increased, there have also been advances in perioperative patient care. For example, Buess initially reported an average 8.7-day hospitalization after TEM, a figure foreign to most modern surgeons whose patients often spend 0 to 2 nights in the hospital after TEM. ${ }^{3}$ Although initially described primarily as a modality for excision of rectal adenomas, resection of cancer using the TEM technique was also reported in the earliest series. ${ }^{2,4}$ We will discuss in some detail benign and malignant indications for TES.

\section{Development of New Platforms}

The TEM platform developed by Buess and produced by Richard Wolf was, for many years, the only one available for TES. It consists of the previously described $40-\mathrm{mm}$ diameter proctoscope, which is available in 12 and $20 \mathrm{~cm}$ lengths, including both blunt and beveled ends. Within the sealing faceplate, there is an optics port and ports for instruments and suction. Instruments are generally angled to prevent collisions within the narrow scope. The advantage of the system has been that it is all inclusive with insufflator, light source, suction, and instrumentation available from the same vendor. A support arm allows fixation to the operating table and, thus, no assistant is required to hold optics or camera. The closed system insufflator regulates instillation of carbon dioxide as the surgeon increases suction or irrigation, allowing for the maintenance of rectal distention and continuous exposure. A limitation of this system is that it requires the pathology to always be "down," thus requiring careful patient positioning. Although the Richard Wolf

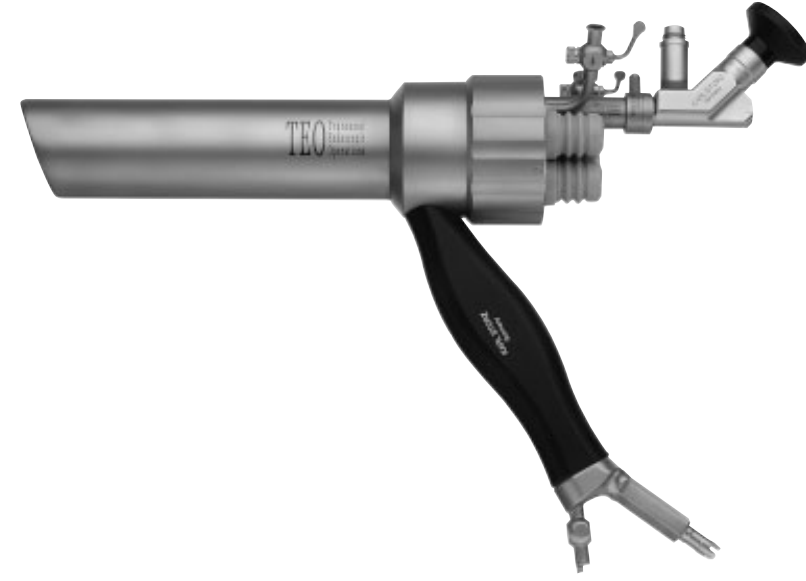

Fig. 2 The TEO proctoscope is similar in design to the TEM scope and consists of the beveled proctoscope itself, a sealing faceplate, and attachments for insufflation, suction, and instrument ports. (2015 Photo Courtesy of KARL STORZ Endoscopy-America, Inc. Used with permission.)

equipment continues to be widely utilized, its cost has been seen as prohibitive for many centers. Karl Storz introduced the transanal endoscopic operation (TEO) system, which is very similar to the Richard Wolf equipment. Both consist of a $40-\mathrm{mm}$ proctoscope, faceplate, support arm, and angled instruments, but TEO offers scope lengths of 7.5, 15, and $20 \mathrm{~cm}$ and an option to use a conventional $5-\mathrm{mm}$ laparoscope (-Fig. 2).

\section{Transanal Minimally Invasive Surgery}

First described in 2010, the transanal minimally invasive surgery (TAMIS) approach took advantage of the development of single-incision laparoscopic surgery ports to increase the access to and decrease the cost of TES..$^{5}$ Instead of a purpose-designed proctoscope, a commercially available single-incision laparoscopic surgery port is inserted in the anus. Approved devices for transanal access include the SILS Port (Covidien, Mansfield, MA) and GelPOINT Path (Applied Medical, Rancho Santa Margarita, CA). A conventional laparoscope and instruments can be used. In addition to substantial cost savings, the TAMIS approach offers more rapid setup time compared with TEM. Also, port insertion and assembly require a fraction of the time of other systems. A key limitation to TAMIS is the increased difficulty in accessing the distal rectum, as the access port obscures the distal several centimeters, and so these lesions must necessarily be approached with traditional TAE. In addition, exposure of the operative field depends on adequate insufflation, given the lack of a large rigid scope providing stable exposure. Comparisons between TEM and TAMIS have been limited, but one small ex vivo study suggested that surgeons unfamiliar with either technique were able to perform suturing tasks more quickly and easily with TEM than with TAMIS and subjectively preferred TEM. ${ }^{6}$ Further evolution of this technique has included the implementation of robotic TAMIS, although increased costs may overshadow the benefits. ${ }^{7}$ Also, there is a growing body of research utilizing TAMIS techniques to 
perform transanal TME; this approach is detailed in a separate section of this issue. ${ }^{8}$

\section{Benign Indications and Outcomes}

Since the first report of a TEM outcomes registry in the United States, there have been a wide variety of indications for the application of this technology. In addition to a large number of resections for carcinomas and adenomas, this initial report by Dr. Lee Smith and others included treatment of carcinoids, strictures, solitary rectal ulcers, prolapse, endometriomas, and enterovaginal fistulas. ${ }^{9}$ As the risk of metastases from small carcinoid tumors is low, local excision via TES may be indicated for those less than $2 \mathrm{~cm}$ in the absence of imaging suggesting metastases. A single-institution series reported no recurrence at a mean follow-up of 70.6 months after TEM resection of 27 small (mean $0.91 \mathrm{~cm}$ ) rectal carcinoid tumors. ${ }^{10}$ Another single-institution series of TEM for small (mean $0.96 \mathrm{~cm}$ ) rectal neuroendocrine tumors also showed promising results. After a median of 3 years of follow-up, 0/59 patients had evidence of recurrence and there was no reported morbidity. ${ }^{11}$ TEM is also a valuable tool for the resection of a prior endoscopic polypectomy site when the polyp is found to contain carcinoid. A single American series showed no carcinoid recurrence in 24 patients, the majority of who underwent TEM for incomplete endoscopic polypectomy. ${ }^{12}$ Case reports and small series have been published discussing the use of TEM or TEO for a wide variety of other benign indications, including excision of tailgut cysts, drainage of pelvic abscess, treatment of benign rectal stenosis, and repair of rectourethral fistula or rectovaginal fistula. ${ }^{13-16}$

\section{Transanal Endoscopic Surgery for Adenomas}

Resection of large, sessile, or recurrent rectal adenomas, or those rectal adenomas that are otherwise endoscopically unresectable, is one of the most widely used applications of TES. For many adenomas, TES has supplanted standard TAE, as TES offers better outcomes in several retrospective trials. ${ }^{17,18}$ These studies have shown the two modalities to have similar complication rates, but TES to be superior for outcomes such as negative margins, specimen fragmentation, and recurrence. In a representative, single-institution review of 259 patients, negative resection margin rate was only $50 \%$ after TAE compared with $88 \%$ after TEM. Specimen fragmentation was $1.4 \%$ after TEM and $23.8 \%$ after TAE and cumulative local recurrence at 5 years was calculated to be $6.1 \%$ after TEM compared with $28.7 \%$ after TAE. ${ }^{19}$ Multiple large series including hundreds of patients have reported recurrence rates after TES for benign polyps ranging from 4 to $7.6 \%{ }^{19-25}$

\section{Indications and Outcomes for Transanal Endoscopic Surgery in Malignancy}

Initial adoption of TEM was based on historical experience with TAE. A meta-analysis of six series comparing TAE to TEM for malignancy demonstrated similar complication rates between the two modalities, but improved rates of negative margins, specimen fragmentation, and local recurrence when TEM was utilized. ${ }^{26}$ Comparison of recurrence between these two modalities may be biased as distal rectal tumors, accessible only by TAE, have been shown to be independently associated with higher rates of recurrence. ${ }^{17}$ With the introduction of the newer TAMIS approach, these favorable margin and specimen fragmentation outcomes have been preserved, suggesting that recurrence rates should be comparable between TEM and TAMIS for malignancy. ${ }^{5}$

Neoadjuvant radiotherapy followed by TME should still be considered the gold standard treatment for oncologic outcomes in rectal cancer, yielding local recurrence rates of less than $1 \%$ and overall survival of $65 \%$ in stage I disease at 12 -year follow-up of the Dutch TME trial. ${ }^{27}$ These impressive oncologic results must be balanced against long-term quality-of-life concerns in surviving patients. At 5 years of follow-up in the same study population, $14 \%$ of irradiated patients had daily fecal incontinence, 52\% reported bowel dysfunction impacting activities outside the house, and 57\% wore pads due to urinary incontinence. Stomas were present in 235/708 (33\%) of patients in this study group. ${ }^{28}$ Outcomes from TES should be considered with these radical resection results in mind.

Current guidelines for local excision of rectal cancer, including the use of TES, are based on long-term survival and outcome data discussed below. National guidelines recommend TAE of only those T1N0 rectal cancers that meet the following criteria: $<30 \%$ circumference of bowel, $<3 \mathrm{~cm}$ in size, $>3 \mathrm{~mm}$ margins, within $8 \mathrm{~cm}$ of anal verge, no negative pathologic features such as lymphovascular or perineural invasion, and tumors that are well to moderately differentiated. ${ }^{29}$ European guidelines suggest that TEM be the procedure of choice for early (T1) rectal cancer without adverse pathologic features because of favorable outcomes including lower rates of positive resection margins and lower rates of local recurrence compared with traditional TAE. $^{30,31}$ As the development of TES techniques has made many rectal tumors previously inaccessible to TAE amenable to local resection, some authors have advocated expanding the criteria for local resection to include any locally resectable T1 rectal malignancy with good pathologic features. Also, as we will discuss later, the addition of adjuvant or neoadjuvant chemotherapy and radiation has led to several trials evaluating local excision for $\mathrm{T} 1$ tumors with adverse histologic features and T2NO tumors. ${ }^{32}$

\section{TEM for T1NO}

The only prospective randomized study comparing TEM to standard oncologic resection for T1N0 rectal cancer randomized 50 patients staged by ultrasound to TEM or anterior resection. ${ }^{33}$ Five-year local recurrence and overall survival were equivalent with one local recurrence in the TEM group and one death due to distant metastases in the radical resection group. Hospital length of stay, blood loss, operative time, and overall early morbidity favored TEM.

A recent, large meta-analysis of 12 observational studies comparing local resection to radical resection in T1N0 rectal cancer illustrates the difficulty currently faced when 
analyzing outcomes of TES for early rectal cancer. ${ }^{34}$ These studies included 2,802 patients whose outcomes could be extracted for overall survival and found worse overall 5-year survival for local resection including TEM and TAE. However, when the TEM subgroup was separately evaluated, there was no difference in overall survival compared with radical resection. It is important to note that within this group, many patients with local recurrence underwent subsequent radical resection. There was increased local recurrence for all locally resected patients (including TEM) compared with radical resection ( $R R, 2.36$; 95\% confidence interval $[C I]$, 1.64-3.39, $p<0.00001$ ), but local resection was protective against perioperative mortality, postoperative complications, and permanent ostomy. The authors noted several potential sources for bias in these studies: a selection bias as surgeons tend to offer more comorbid patients local resection and an over-representation of low rectal cancers in the local excision group. As low rectal cancers have poorer prognosis, the authors suggested that both of the sources of bias skew toward a less favorable outcome for local resection. This may also explain the favorable outcomes of TEM over TAE, as TEM is more suited to more proximal rectal cancers. Another recent meta-analysis comparing only TEM to TME found similar results with higher risk of local recurrence for patients undergoing TEM (odds ratio $=4.62$, 95\% CI: 2.03-10.53, $p=0.0003$ ) but no significant difference in overall survival or distant metastasis. ${ }^{35}$ Single-institution reviews of oncologic outcomes after TEM for T1N0 cancers have shown recurrence rates of 0 to $24 \%$ (-Table 1 ).

\section{TEM for T2NO}

Recurrence rates for T2N0 rectal cancer treated by local resection have been significantly higher than those for $\mathrm{T} 1$ lesions in small series, often reporting outcomes for patients who declined TME or were medically unfit. In these series, with unstandardized chemotherapy and radiation therapy protocols, local recurrence was 4.7 to $26 \% .^{25,36,37}$ Limited clinical trials have shown more promising results. The Urbino trial was a study of long-term oncologic outcomes for 70 patients with T2N0 rectal cancer randomized to TEM or laparoscopic resection (low anterior resection or abdominal perineal resection) following neoadjuvant chemoradiation. ${ }^{38}$ After a median of 84 months of follow-up, the local recurrence rate was 5.7\% after TEM and $2.8 \%$ after laparoscopic resection. There was no difference in disease-free survival between the two groups ( $94 \%$ DF for both TEM and laparoscopic resection), and a single patient in each group developed distant metastases. There was no significant difference in overall complication rate between the two groups, but there was a shorter hospital stay for patients undergoing TEM compared with laparoscopic resection ( 3 vs. 7 days, $p<0.001$ ). Multiple studies by the same group of authors have shown similar outcomes. ${ }^{39-41}$ Rates of recurrence of T2 rectal cancers treated by TEM are shown in - Table 2.

The American College of Surgeons Oncology Group (ACOSOG) Z6041 trial was designed as a prospective, singlearmed, multicenter evaluation of the complications and oncologic outcomes of neoadjuvant chemoradiation followed by local excision for clinically staged T2N0 rectal cancer. ${ }^{42}$ In a recent

Table 1 Recurrence rate of T1 rectal cancer following TES

\begin{tabular}{|c|c|c|c|c|c|}
\hline Series & Year & Platform & Number & Recurrence & Follow-up: mean months \\
\hline Winde et $\mathrm{al}^{33}$ & 1996 & TEM & 24 & $4.1 \%$ & 40.9 \\
\hline Heintz et al ${ }^{62}$ & 1998 & TEM & 44 & $4.5 \%$ & 52 \\
\hline Lee et $\mathrm{al}^{63}$ & 2003 & TEM & 25 & $4.1 \%$ & 31 \\
\hline Floyd and Saclarides ${ }^{64}$ & 2006 & TEM & 53 & $7.5 \%$ & 34.1 \\
\hline Ganai et al ${ }^{65}$ & 2006 & TEM & 21 & $19 \%$ & 43 \\
\hline Bretagnol et $\mathrm{al}^{20}$ & 2007 & TEM & 31 & $9.7 \%$ & 34 \\
\hline Baatrup et al ${ }^{36}$ & 2009 & TEM & 72 & $13 \%$ & - \\
\hline Jeong et $\mathrm{al}^{66}$ & 2009 & TEM & 17 & $0 \%$ & 37 \\
\hline Allaix et al $^{67}$ & 2009 & TEM & 38 & $0 \%$ & 60 \\
\hline De Graaf et al ${ }^{68}$ & 2009 & TEM & 80 & $24 \%$ & 42 \\
\hline Palma et $\mathrm{al}^{69}$ & 2009 & TEM & 34 & $5.9 \%$ & 86.5 \\
\hline Tsai et $\mathrm{al}^{25}$ & 2010 & TEM & 51 & $9.8 \%$ & 53.9 \\
\hline Doornebosch et al ${ }^{70}$ & 2010 & TEM & 88 & 20.5 & - \\
\hline Steinhagen et al ${ }^{71}$ & 2011 & TEM & 12 & $0 \%$ & 33 \\
\hline Ramirez et $\mathrm{al}^{72}$ & 2011 & TEM & 53 & $7.5 \%$ & 71 \\
\hline Lezoche et $\mathrm{al}^{37}$ & 2011 & TEM & 51 & $0 \%$ & 97 \\
\hline Stipa et $\mathrm{al}^{73}$ & 2012 & TEM & 86 & $11.6 \%$ & 85 \\
\hline Albert et al ${ }^{46}$ & 2013 & TAMIS & 16 & $6.3 \%$ & 20.9 \\
\hline
\end{tabular}

Abbreviations: TAMIS, transanal minimally invasive surgery; TEM, transanal endoscopic microsurgery; TES, transanal endoscopic surgery. 
Table 2 Recurrence rate of T2 rectal cancer following TES

\begin{tabular}{|c|c|c|c|c|c|c|}
\hline Series & Year & Platform & Number & Chemoradiation & Recurrence & Follow-up: months \\
\hline Endreseth et al ${ }^{74}$ & 2005 & TEM & 5 & None & $20 \%$ & 24 \\
\hline Ganai et al ${ }^{65}$ & 2006 & TEM & 4 & Adjuvant & $50 \%$ & 44 \\
\hline Jeong et al ${ }^{66}$ & 2009 & TEM & 6 & Adjuvant & $16.7 \%$ & 37 \\
\hline Tsai et $\mathrm{al}^{25}$ & 2010 & TEM & 17 & Selective & $23.5 \%$ & 42.8 \\
\hline Lezoche et $\mathrm{al}^{37}$ & 2011 & TEM & 84 & Neoadjuvant & $4.7 \%$ & 97 \\
\hline Amann et $\mathrm{al}^{75}$ & 2015 & TEM & 53 & Variable & $20.8 \%$ & 33.9 \\
\hline
\end{tabular}

Abbreviations: TEM, transanal endoscopic microsurgery; TES, transanal endoscopic surgery.

report of initial results, the study's authors analyzed all 77 patients who completed treatment according to study protocols for pathologic response and complications. Pathologic complete response was noted in $44 \%$ of patients, and a single patient had positive margins. There were grade $\geq 3$ complications reported in 33 out of 77 patients (43\%) attributable to chemoradiotherapy (CRT) and, overall, 45 out of 77 patients (58\%) reported any complication related to surgery. Oncologic results of this trial are to be reported after a 3-year follow-up period.

There has been an increasing interest in the use of TEM as a mechanism for evaluating pathologic response to neoadjuvant chemoradiation and as an option for rectumpreserving treatment of select early-stage rectal cancer. The recently published CARTS study followed up 55 T13NO rectal cancer patients after chemoradiation. ${ }^{43}$ Those with significant tumor downsizing underwent TEM, and the 30 out of 55 (55\%) patients with ypT0-1 on TEM specimen were considered to have completed surgical therapy. After a median follow-up of 17 months, only 1 of these 30 patients had developed local recurrence and underwent APR. An earlier report of 89 patients with sessile $\mathrm{T} 1, \mathrm{~T} 2$, or $\mathrm{T} 3$ rectal tumors treated by a similar protocol also offered promising results. ${ }^{44}$ Despite unreliable adherence to radiation protocols, these authors were able to demonstrate a $10 \%$ local recurrence rate at 2 years in those patients with ypT0-1 tumors in local excision specimen after neoadjuvant chemoradiation or short-course radiation alone. These authors performed local excision with TEM or traditional TAE.

A significant concern raised about protocols like the ones described previously is the outcome of a patient who underwent neoadjuvant chemoradiation and TEM resection of node-negative rectal cancer but declines definitive TME offered by his or her surgeon based on high-risk pathologic findings in the local resection specimen. Two trials reporting these outcomes have had sobering results. In the first, 8 out of $18(44.4 \%)$ patients who declined completion TME suffered local recurrence and one additional patient had a distant metastasis without local recurrence. ${ }^{44}$ A more recent study reports 12 out of 36 (33.3\%) patients with local recurrence. ${ }^{45}$ Of these 12, only 8 patients were candidates for completion TME, but only 1 of 8 patients was able to achieve negative circumferential resection margin.

The limited reports of TAMIS outcomes are similar to those for TEM for both benign adenomas and early cancers.
One center reported $4 \%$ recurrence at 18 months with no long-term complications. ${ }^{46}$

\section{Functional Outcomes}

The 4-cm diameter TEM scope has been associated with varying degrees of fecal incontinence. One early singleinstitution review revealed a $37 \%$ rate of worsened continence after TEM. ${ }^{47}$ Incontinence rates appeared to persist over time in this study. Another, more recent, single-institution review of 222 patients showed that while there were statistically significant declines in manometrically measured anal baseline pressure and voluntary contraction pressure at 1 and 4 months after TEM, there were no changes in clinical fecal incontinence scores. ${ }^{48}$ Several studies have reported similar outcomes with no long-term change or improvement in postoperative clinical fecal incontinence scores compared with baseline. ${ }^{49-53}$ Single-institution reviews of functional outcomes after TAMIS have also reported preserved or improved fecal continence and overall quality of life. ${ }^{54-56}$

\section{Technical Issues}

\section{Lesion Site}

As mentioned previously, patient positioning is of critical importance in TEM and must also be considered for TAMIS approaches. Therefore, the surgeon must perform rigid proctoscopy prior to resection both to determine the level of the lesion within the rectum and to precisely characterize its orientation. As the TEM proctoscope is beveled downward, the patient must be positioned so that the lesion is down relative to the scope, for example, the patient must be in prone position for an anterior lesion or right side down for a right lateral lesion. Even after the patient is properly positioned, the surgeon may have to reposition the scope throughout dissection to keep the operative site within the center of the field of view. Because there is no orientation to the single-site access ports used in TAMIS, and the angled laparoscope allows 360-degree visualization, there is no absolute requirement that the patient be positioned with the lesion down. Although Drs. Atallah and Larach, in their original description of TAMIS, did position the patient so that the lesion was down, their more recent publications have advocated for high lithotomy position for all lesion 
locations. $^{46}$ Other authors have recommended lithotomy positioning for all distal and mid rectal lesions, but favor prone positioning for anterior proximal lesions. ${ }^{57}$ Another key concern is the risk of perforation into the abdominal cavity for more proximal lesions. As resections for malignancy are performed as full-thickness excisions, the surgeon must have a high degree of suspicion for detecting a fullthickness injury, especially for lesions located anteriorly, since the peritoneal reflection dips down more substantially anteriorly. These proctotomies can usually be closed adequately via the TES approach, but larger injuries may require an abdominal approach to repair. Depending on the comfort level and skill of the surgeon, these can usually be performed in a minimally invasive fashion. Such entries into the peritoneum usually mandate a longer period of in-hospital observation.

While TES offers acceptable local recurrence and longterm survival outcomes with minimal morbidity for many low rectal cancers, some lesions may not be technically resectable due to location, size, or other factors. Traditional contraindications for TAE have included tumors $>30 \%$ of the luminal circumference, $>3 \mathrm{~cm}$ in size, or $>8 \mathrm{~cm}$ from the anal verge. ${ }^{29}$ A recent retrospective review of a single institution's prospective database defined technically challenging lesions as those $\geq 5 \mathrm{~cm}$ in diameter, involving $\geq 50 \%$ of the lumen, or $\geq 10 \mathrm{~cm}$ from the anal verge. ${ }^{58}$ These authors found that lesions possessing one of these technically challenging attributes had equivalent outcomes for many factors including local recurrence, but tumors with two or more of these attributes had significantly worse outcomes, including more local recurrence, peritoneal perforation, tumor fragmentation, rectal stenosis, and incomplete resection. As with many new surgical techniques, factors once thought to be absolute contraindications are rendered merely relative ones, based on experience and skill.

\section{Cost}

Published data on cost of surgical equipment are notoriously variable and difficult to compare, but several reports have offered insight into the systems discussed. Initial costs of specialized TEM equipment are seen as barrier to entry for many centers and may be upward of US $\$ 80,000$. However, it has also been noted that, even after accounting for initial equipment costs, the total cost of TEM resection of a distal rectal tumor is dramatically lower than that of traditional open resection for matched lesions due to shorter length of stay, fewer complications, and the absence of a diverting stoma requiring closure. ${ }^{59,60}$ Several authors have commented that the US\$335 to 650 cost of the single-site ports used for TAMIS is less than the cost of the disposable tubing and seals used with TEM. 5,46,61

\section{Conclusion}

TES continues to be a rapidly evolving field, even three decades after it was first described. With better functional outcomes than radical resection and equivalent oncologic results in properly selected patients, surgeons will continue to explore the limits of these techniques to improve patient care.

\section{References}

1 Buess G, Theiss R, Hutterer F, et al. Transanal endoscopic surgery of the rectum - testing a new method in animal experiments [in German]. Leber Magen Darm 1983;13(2):73-77

2 Buess G, Theiss R, Günther M, Hutterer F, Pichlmaier H. Endoscopic surgery in the rectum. Endoscopy 1985;17(1):31-35

3 Buess G, Kipfmüller K, Ibald R, et al. Clinical results of transanal endoscopic microsurgery. Surg Endosc 1988;2(4):245-250

4 Buess G, Kipfmüller K, Naruhn M, Braunstein S, Junginger T. Endoscopic microsurgery of rectal tumors. Endoscopy 1987;19 (Suppl 1):38-42

5 Atallah S, Albert M, Larach S. Transanal minimally invasive surgery: a giant leap forward. Surg Endosc 2010;24(9):2200-2205

6 Rimonda R, Arezzo A, Arolfo S, Salvai A, Morino M. TransAnal minimally invasive surgery (TAMIS) with SILS port versus transanal endoscopic microsurgery (TEM): A comparative experimental study. Surg Endosc 2013;27(10):3762-3768

7 Hompes R, Rauh SM, Ris F, Tuynman JB, Mortensen NJ. Robotic transanal minimally invasive surgery for local excision of rectal neoplasms. Br J Surg 2014;101(5):578-581

8 Deijen CL, Velthuis S, Tsai A, et al. COLOR III: a multicentre randomised clinical trial comparing transanal TME versus laparoscopic TME for mid and low rectal cancer. Surg Endosc 2016; 30(8):3210-3215

9 Smith LE, Ko ST, Saclarides T, Caushaj P, Orkin BA, Khanduja KS. Transanal endoscopic microsurgery. Initial registry results. Dis Colon Rectum 1996;39, (10 Suppl):S79-S84

10 Kinoshita T, Kanehira E, Omura K, Tomori T, Yamada H. Transanal endoscopic microsurgery in the treatment of rectal carcinoid tumor. Surg Endosc 2007;21(6):970-974

11 Chen WJ, Wu N, Zhou JL, Lin GL, Qiu HZ. Full-thickness excision using transanal endoscopic microsurgery for treatment of rectal neuroendocrine tumors. World J Gastroenterol 2015;21(30): 9142-9149

12 Kumar AS, Sidani SM, Kolli K, et al. Transanal endoscopic microsurgery for rectal carcinoids: the largest reported United States experience. Colorectal Dis 2012;14(5):562-566

13 Bochove-Overgaauw DM, Beerlage HP, Bosscha K, Gelderman WA. Transanal endoscopic microsurgery for correction of rectourethral fistulae. J Endourol 2006;20(12):1087-1090

14 Duek SD, Gilshtein H, Khoury W. Transanal endoscopic microsurgery: also for the treatment of retrorectal tumors. Minim Invasive Ther Allied Technol 2014;23(1):28-31

15 Pigalarga R, Patel NM, Rezac C. Transanal endoscopic microsurgery-assisted rectal advancement flap is a viable option for iatrogenic rectourethral fistula repair: a case report. Tech Coloproctol 2011;15(2):209-211

16 Serra-Aracil X, Mora-Lopez L, Alcantara-Moral M, CorrederaCantarin C, Gomez-Diaz C, Navarro-Soto S. Atypical indications for transanal endoscopic microsurgery to avoid major surgery. Tech Coloproctol 2014;18(2):157-164

17 Christoforidis D, Cho HM, Dixon MR, Mellgren AF, Madoff RD, Finne CO. Transanal endoscopic microsurgery versus conventional transanal excision for patients with early rectal cancer. Ann Surg 2009;249(5):776-782

18 Moore JS, Cataldo PA, Osler T, Hyman NH. Transanal endoscopic microsurgery is more effective than traditional transanal excision for resection of rectal masses. Dis Colon Rectum 2008;51(7): 1026-1030, discussion 1030-1031

19 de Graaf EJ, Burger JW, van Ijsseldijk AL, Tetteroo GW, Dawson I, Hop WC. Transanal endoscopic microsurgery is superior to transanal excision of rectal adenomas. Colorectal Dis 2011; 13(7):762-767 
20 Bretagnol F, Merrie A, George B, Warren BF, Mortensen NJ. Local excision of rectal tumours by transanal endoscopic microsurgery. Br J Surg 2007;94(5):627-633

21 de Graaf EJ, Doornebosch PG, Tetteroo GW, Geldof H, Hop WC. Transanal endoscopic microsurgery is feasible for adenomas throughout the entire rectum: a prospective study. Dis Colon Rectum 2009;52(6):1107-1113

22 Guerrieri M, Baldarelli M, de Sanctis A, Campagnacci R, Rimini M, Lezoche $E$. Treatment of rectal adenomas by transanal endoscopic microsurgery: 15 years' experience. Surg Endosc 2010;24(2): 445-449

23 Ramirez JM, Aguilella V, Gracia JA, et al. Local full-thickness excision as first line treatment for sessile rectal adenomas: long-term results. Ann Surg 2009;249(2):225-228

24 Said S, Stippel D. Transanal endoscopic microsurgery in large, sessile adenomas of the rectum. A 10-year experience. Surg Endosc 1995;9(10):1106-1112

25 Tsai BM, Finne CO, Nordenstam JF, Christoforidis D, Madoff RD, Mellgren A. Transanal endoscopic microsurgery resection of rectal tumors: outcomes and recommendations. Dis Colon Rectum 2010;53(1):16-23

26 Clancy C, Burke JP, Albert MR, O'Connell PR, Winter DC. Transanal endoscopic microsurgery versus standard transanal excision for the removal of rectal neoplasms: a systematic review and metaanalysis. Dis Colon Rectum 2015;58(2):254-261

27 van Gijn W, Marijnen CA, Nagtegaal ID, et al; Dutch Colorectal Cancer Group. Preoperative radiotherapy combined with total mesorectal excision for resectable rectal cancer: 12-year followup of the multicentre, randomised controlled TME trial. Lancet Oncol 2011;12(6):575-582

28 Peeters KC, van de Velde CJ, Leer JW, et al. Late side effects of shortcourse preoperative radiotherapy combined with total mesorectal excision for rectal cancer: increased bowel dysfunction in irradiated patients-a Dutch colorectal cancer group study. J Clin Oncol 2005;23(25):6199-6206

29 NCCN guidelines version 3.2015 rectal cancer. NCCN Clinical Practice Guidelines in Oncology. Web site: http://www.nccn. org/professionals/physician_gls/f_guidelines.asp\#rectal. Updated 2015. Accessed 09/07, 2015

30 Arezzo A, Bianco F, Agresta F, et al; Italian Society of Colorectal Surgery. Practice parameters for early rectal cancer management: Italian Society of Colorectal Surgery (Società Italiana di Chirurgia Colo-Rettale; SICCR) guidelines. Tech Coloproctol 2015;19(10): 587-593

31 Morino M, Risio M, Bach S, et al; European Association for Endoscopic Surgery; European Society of Coloproctology. Early rectal cancer: the European Association for Endoscopic Surgery (EAES) clinical consensus conference. Surg Endosc 2015;29(4): 755-773

32 Cataldo PA. Local excision of rectal cancer. In: Beck D, Roberts P, Saclarides T, Senagore A, Stamos M, Wexner S, eds. The ASCRS Textbook of Colon and Rectal Surgery. New York: Springer, 2011: 731

33 Winde G, Nottberg H, Keller R, Schmid KW, Bünte H. Surgical cure for early rectal carcinomas (T1). Transanal endoscopic microsurgery vs. anterior resection. Dis Colon Rectum 1996;39(9): 969-976

34 Kidane B, Chadi SA, Kanters S, Colquhoun PH, Ott MC. Local resection compared with radical resection in the treatment of T1N0M0 rectal adenocarcinoma: a systematic review and metaanalysis. Dis Colon Rectum 2015;58(1):122-140

35 Lu JY, Lin GL, Qiu HZ, Xiao Y, Wu B, Zhou JL. Comparison of transanal endoscopic microsurgery and total mesorectal excision in the treatment of T1 rectal cancer: A meta-analysis. PLoS One 2015;10(10):e0141427

36 Baatrup G, Breum B, Qvist N, et al. Transanal endoscopic microsurgery in 143 consecutive patients with rectal adenocarcinoma: results from a Danish multicenter study. Colorectal Dis 2009; 11(3):270-275

37 Lezoche G, Guerrieri M, Baldarelli M, et al. Transanal endoscopic microsurgery for 135 patients with small nonadvanced low rectal cancer (iT1-iT2, iN0): short- and long-term results. Surg Endosc 2011;25(4):1222-1229

38 Lezoche G, Baldarelli M, Guerrieri M, et al. A prospective randomized study with a 5-year minimum follow-up evaluation of transanal endoscopic microsurgery versus laparoscopic total mesorectal excision after neoadjuvant therapy. Surg Endosc 2008;22(2):352-358

39 Lezoche E, Guerrieri M, Paganini AM, Feliciotti F. Long-term results of patients with pT2 rectal cancer treated with radiotherapy and transanal endoscopic microsurgical excision. World J Surg 2002;26(9):1170-1174

40 Lezoche E, Guerrieri M, Paganini AM, et al. Transanal endoscopic versus total mesorectal laparoscopic resections of T2-NO low rectal cancers after neoadjuvant treatment: a prospective randomized trial with a 3-years minimum follow-up period. Surg Endosc 2005;19(6):751-756

41 Lezoche E, Baldarelli M, Lezoche G, Paganini AM, Gesuita R, Guerrieri M. Randomized clinical trial of endoluminal locoregional resection versus laparoscopic total mesorectal excision for T2 rectal cancer after neoadjuvant therapy. Br J Surg 2012;99(9): 1211-1218

42 Garcia-Aguilar J, Shi Q, Thomas CR Jr, et al. A phase II trial of neoadjuvant chemoradiation and local excision for T2N0 rectal cancer: preliminary results of the ACOSOG Z6041 trial. Ann Surg Oncol 2012;19(2):384-391

43 Verseveld M, de Graaf EJ, Verhoef C, et al; CARTS Study Group. Chemoradiation therapy for rectal cancer in the distal rectum followed by organ-sparing transanal endoscopic microsurgery (CARTS study). Br J Surg 2015;102(7):853-860

44 Bujko K, Richter P, Smith FM, et al. Preoperative radiotherapy and local excision of rectal cancer with immediate radical re-operation for poor responders: a prospective multicentre study. Radiother Oncol 2013;106(2):198-205

45 Perez RO, Habr-Gama A, São Julião GP, et al. Transanal endoscopic microsurgery (TEM) following neoadjuvant chemoradiation for rectal cancer: outcomes of salvage resection for local recurrence. Ann Surg Oncol 2016;23(4):1143-1148

46 Albert MR, Atallah SB, deBeche-Adams TC, Izfar S, Larach SW. Transanal minimally invasive surgery (TAMIS) for local excision of benign neoplasms and early-stage rectal cancer: efficacy and outcomes in the first 50 patients. Dis Colon Rectum 2013;56(3): 301-307

47 Dafnis G, Påhlman L, Raab Y, Gustafsson UM, Graf W. Transanal endoscopic microsurgery: clinical and functional results. Colorectal Dis 2004;6(5):336-342

48 Mora López L, Serra Aracil X, Hermoso Bosch J, Rebasa P, Navarro Soto S. Study of anorectal function after transanal endoscopic surgery. Int J Surg 2015;13:142-147

49 Cataldo PA, O'Brien S, Osler T. Transanal endoscopic microsurgery: a prospective evaluation of functional results. Dis Colon Rectum 2005;48(7):1366-1371

50 Allaix ME, Rebecchi F, Giaccone C, Mistrangelo M, Morino M. Longterm functional results and quality of life after transanal endoscopic microsurgery. Br J Surg 2011;98(11):1635-1643

51 Hompes R, Ashraf SQ Gosselink MP, et al. Evaluation of quality of life and function at 1 year after transanal endoscopic microsurgery. Colorectal Dis 2015;17(2):054-061

52 Planting A, Phang PT, Raval MJ, Brown CJ. Transanal endoscopic microsurgery: impact on fecal incontinence and quality of life. Can J Surg 2013;56(4):243-248

53 Barendse RM, Oors JM, de Graaf EJ, et al. The effect of endoscopic mucosal resection and transanal endoscopic microsurgery on anorectal function. Colorectal Dis 2013;15(9):e534-e541 
54 Karakayali FY, Tezcaner T, Moray G. Anorectal function and outcomes after transanal minimally invasive surgery for rectal tumors. J Minim Access Surg 2015;11(4):257-262

55 Schiphorst AH, Langenhoff BS, Maring J, Pronk A, Zimmerman DD. Transanal minimally invasive surgery: initial experience and shortterm functional results. Dis Colon Rectum 2014;57(8):927-932

56 Verseveld M, Barendse RM, Gosselink MP, Verhoef C, de Graaf EJ, Doornebosch PG. Transanal minimally invasive surgery: impact on quality of life and functional outcome. Surg Endosc 2016; 30(3):1184-1187

57 Keller DS, Haas EM. Transanal minimally invasive surgery: state of the art. J Gastrointest Surg 2016;20(2):463-469

58 Saget A, Maggiori L, Petrucciani N, Ferron M, Panis Y. Is there a limit to transanal endoscopic surgery? A comparative study between standard and technically challenging indications among 168 consecutive patients. Colorectal Dis 2015;17(7):0155-0160

59 Maslekar S, Pillinger SH, Sharma A, Taylor A, Monson JR. Cost analysis of transanal endoscopic microsurgery for rectal tumours. Colorectal Dis 2007;9(3):229-234

60 Farmer KC, Wale R, Winnett J, Cunningham I, Grossberg P, Polglase A. Transanal endoscopic microsurgery: the first 50 cases. ANZ J Surg 2002;72(12):854-856

61 Barendse RM, Doornebosch PG, Bemelman WA, Fockens P, Dekker E, de Graaf EJ. Transanal employment of single access ports is feasible for rectal surgery. Ann Surg 2012;256(6):1030-1033

62 Heintz A, Mörschel M, Junginger T. Comparison of results after transanal endoscopic microsurgery and radical resection for T1 carcinoma of the rectum. Surg Endosc 1998;12(9):1145-1148

63 Lee W, Lee D, Choi S, Chun H. Transanal endoscopic microsurgery and radical surgery for T1 and T2 rectal cancer. Surg Endosc 2003; 17(8):1283-1287

64 Floyd ND, Saclarides TJ. Transanal endoscopic microsurgical resection of pT1 rectal tumors. Dis Colon Rectum 2006;49(2):164-168

65 Ganai S, Kanumuri P, Rao RS, Alexander AI. Local recurrence after transanal endoscopic microsurgery for rectal polyps and early cancers. Ann Surg Oncol 2006;13(4):547-556
66 Jeong WK, Park JW, Choi HS, Chang HJ, Jeong SY. Transanal endoscopic microsurgery for rectal tumors: experience at Korea's National Cancer Center. Surg Endosc 2009;23(11): 2575-2579

67 Allaix ME, Arezzo A, Caldart M, Festa F, Morino M. Transanal endoscopic microsurgery for rectal neoplasms: experience of 300 consecutive cases. Dis Colon Rectum 2009;52(11):1831-1836

68 De Graaf EJ, Doornebosch PG, Tollenaar RA, et al. Transanal endoscopic microsurgery versus total mesorectal excision of $\mathrm{T} 1$ rectal adenocarcinomas with curative intention. Eur J Surg Oncol 2009;35(12):1280-1285

69 Palma P, Horisberger K, Joos A, Rothenhoefer S, Willeke F, Post S. Local excision of early rectal cancer: is transanal endoscopic microsurgery an alternative to radical surgery? Rev Esp Enferm Dig 2009;101(3):172-178

70 Doornebosch PG, Ferenschild FT, de Wilt JH, Dawson I, Tetteroo GW, de Graaf EJ. Treatment of recurrence after transanal endoscopic microsurgery (TEM) for T1 rectal cancer. Dis Colon Rectum 2010;53(9):1234-1239

71 Steinhagen E, Chang G, Guillem JG. Initial experience with transanal endoscopic microsurgery: the need for understanding the limitations. J Gastrointest Surg 2011;15(6):958-962

72 Ramirez JM, Aguilella V, Valencia J, et al. Transanal endoscopic microsurgery for rectal cancer. Long-term oncologic results. Int J Colorectal Dis 2011;26(4):437-443

73 Stipa F, Giaccaglia V, Burza A. Management and outcome of local recurrence following transanal endoscopic microsurgery for rectal cancer. Dis Colon Rectum 2012;55(3):262-269

74 Endreseth BH, Wibe A, Svinsås M, Mårvik R, Myrvold HE. Postoperative morbidity and recurrence after local excision of rectal adenomas and rectal cancer by transanal endoscopic microsurgery. Colorectal Dis 2005;7(2):133-137

75 Amann M, Burghardt J, Stratz C, Buess GF, Modabber A. Transanal endoscopic microsurgery in treatment of small rectal T1 highrisk, T2 and T3 carcinomas combined with radiochemotherapy. Eur Surg 2015;47(5):226-237 